\title{
Deep Network for Content and Context Based Image Retrieval System
}

\author{
Arpana Mahajan, Sanjay Chaudhary
}

\begin{abstract}
The brisk improvement in sight and sound and imaging advancement, the amounts of pictures moved and shared on the web have extended. It prompts to develop the particularly reasonable picture recovery system to satisfy human needs. The substance setting and contain picture recovery structure which recovers the picture subject to the likeness of the huge highlights, for instance, names which are unquestionably not satisfactory to depict the customer's low-level insight for pictures. In this exploration paper lessening this semantic issue of picture recovery is a difficult errand. Presumably the most critical considerations in picture recovery are watchwords, terms or thoughts. Here separated picture highlights from a pre-prepared profound system (RESNET), and utilize that highlights to prepare profound learning classifier. Remaining profound systems make include extraction most effortless and quickest approach to use than some other profound system strategy. In this exploration paper, we portray Image recovery utilizing proposed lingering profound systems.
\end{abstract}

Keywords-Context, Contain, Image retrieval; Residual; Layers, deep network

\section{INTRODUCTION}

The automated improvement drives the cutting edge society to process a lot of images and video accumulation, along these lines, a size of the interactive media database is developed massively. This prompts building up a viable perusing and looking through instruments that skirt the emotional undertaking of manual catchphrase ordering and to make ready for the eager and testing thought of the substance based portrayal of symbolism. All the data about the substance of an image doesn't just get from the image itself rather it originates from different sources as well. Albeit visual properties of the image itself can be viewed as the setting of an image, it doesn't give full data just by it. Because of an enormous number of web images, it is urgent to create procedures to rapidly explore clients to their intriguing images, and image search is one of such methods [4]. Image retrieval is utilized in an enormous assortment of uses, for example, for individual visual data search, photojournalism, and workmanship, web based learning, for medicinal and logical applications among numerous others. The expansion in the quantity of existing images just as in the enhancement of new techniques for image procurement requires perpetually exact image retrieval frameworks [3].

Revised Manuscript Received on September 15, 2019.

* Correspondence Author

Mrs. Arpana Mahajan", "Research Scholar," "Computer

"mahajan.arpana@yahoo.com

Dr. Sanjay Chaudhary", "Research Supervisor," "Computer Engineering," "Madhav University," "Rajasthan, India" "schaudhary00@gmail.com

An uncommon type of context based retrieval is design similitude, otherwise called spatial, auxiliary, or game plan closeness. A definitive objective of image retrieval is to give the clients the office to oversee huge image categorical databases in a programmed, adaptable and proficient way. In this way, image categories based retrieval frameworks ought to be outfitted to help significant level (semantics based) questioning and perusing of images [5]

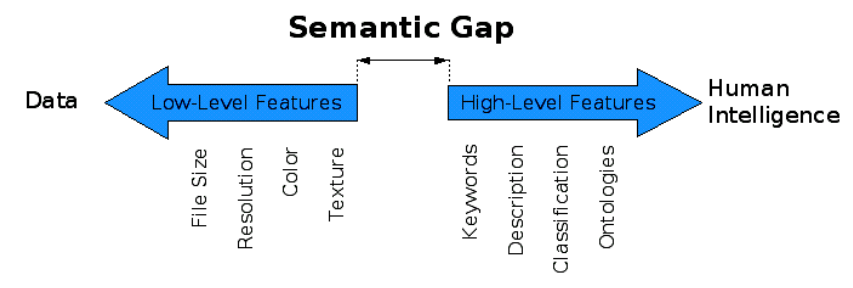

Fig. 1.Retrival System Gap

\section{LITERATURE REVIEW}

From, a long time, the retrieval of content and images has been founded on physically made lists put away as back of the book lists or card records. Most libraries, image files, and video files still utilize these files. In the most recent decades, systems have been created to consequently record huge volumes of content and a portion of these strategies are likewise used to list images based on related content (setting based image retrieval). In the most recent years, image preparing systems have been built up that permit the ordering of images dependent on their visual substance (content-based image retrieval). This area depicts these two distinct ways to deal with image retrieval. In the following subsections, the essential techniques and issues of separately setting based image retrieval and substance based image retrieval will be examined.

\section{A. Context Related Retrieval Approach}

A great deal of data about the substance of an image can emerge out of different sources than the image itself. All data that doesn't originate from the visual properties of the image itself can be viewed as the setting of an image. For instance, where you found an image or the individual who pointed you at it can enlighten a great deal concerning the data showed in the image. In this paper in any case, we utilize the term setting just for the literary data that accompanies an image. Setting based image retrieval can be founded on comments that were physically included for revealing the images (watchwords, portrayals), or on security message that is accidentally available with an image (inscriptions, captions, close by content). 
From these writings, files can be made utilizing standard content retrieval procedures. The comparability between images is then founded on the likeness between the related writings, which thusly is frequently founded on similitude in word use. A significant issue with this methodology is the distinction in word use between reports. Records can talk about a similar subject utilizing various words (synonymy) or utilize similar words portraying various ideas (vagueness). This issue, which likewise happens in full-content retrieval, is known as the reword issue (Oard and Dorr, 1996). It tends to be overwhelmed by utilizing a limited jargon for manual comment (controlled term ordering), however it is extravagant to physically record all images in a huge accumulation.

\section{B. Content Related Retrival System}

Content-based image retrieval (CBIR) utilizing inquiry by model (QBE) has turned out to be mainstream over the most recent couple of years. CBIR frameworks attempt to restore those images that are outwardly most like a model image; closeness is based on a lot of low-level image highlights. Highlights that can be utilized to list images are shading, surface, shape and spatial format. A few examinations exist on what highlights best match human observation (Gargi and Kasturi, 1996; Liu and Picard, 1996), at the same time, mostly in light of the subjectivity included, it is doubtful that such a list of capabilities exists by any means. Another significant issue with content-based ordering is the way that visual likeness doesn't compare to semantic similitude. Along these lines, regardless of whether a list of capabilities existed that matches the human vision, still the recovered images aren't really identified with the model image on a semantic level. This issue is known as the semantic hole and causes current image retrieval frameworks to recover for instance images of ladies in red dresses when the model image was an image of a red vehicle.

\section{Revealing Hidden Semantics}

In the past areas, we saw that one of the serious issues in both setting based image retrieval and content-based image retrieval is the way that the terms1 in an archive (words or low-level image highlights) vary from the semantic content of a report. In any case, this doesn't mean, that the term of a report are absolutely good for nothing. All things considered, people utilize, in addition to other things, a similar arrangement of terms to find the semantics of an archive. Accordingly, we need a strategy that reveals these concealed semantics of a record, or possibly can dismiss diverse term use in related reports.

\section{Proposed SYSTEM}

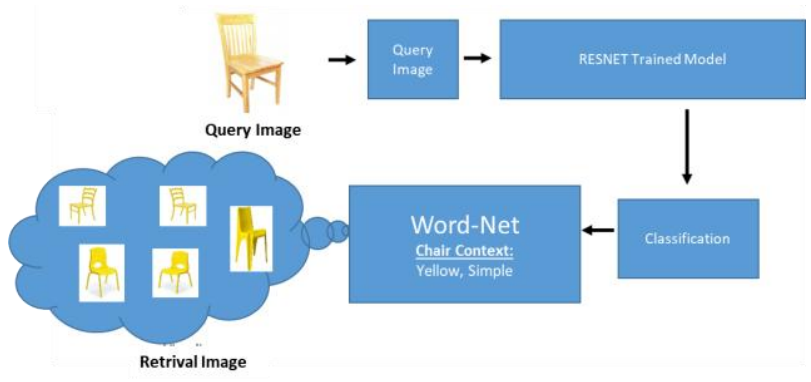

Fig. 2.Proposed System

\section{Input:}

Query Image ' $Q$ ' with associated tags $\mathrm{TG}=\{\operatorname{tg} 1, \operatorname{tg} 2 \ldots \operatorname{tgm}\}$ Output:

Top $\mathrm{N}$ retrieved images form database $\mathrm{DA}=\{\mathrm{Q} 1, \mathrm{Q} 2 \ldots \mathrm{Qn}\}$

$\mathrm{FQ}=$ WordNet Dictionary of query image $\mathrm{Q}$

$\mathrm{FDA}=$ Set of feature vector of $n$ database images $=\{F Q 1$,

FQ2 ....FQn

Hybrid Image Retrieval Algorithm (Q, DT)

\section{BEGIN}

1. For $\mathrm{i}=1$ to $\mathrm{N}$ database images

2. Apply RESNET feature extractor to image Q, to obtain resultant feature vector FDAi.

3. Apply SUPPORT VECTOR MACHINE (SVM) Trainer to FDAi for training and make SUPPORT VECTOR MACHINE (SVM) classification.

4. Apply step (2) on query image Q and obtain FDA, Give FDA to trained classifier to obtain class CQ of the query image Q.

5. Apply WordNet Distance based retrieval System to retrieve query images in query class $\mathrm{CQ}$.

END

\section{A. Feture Learning}
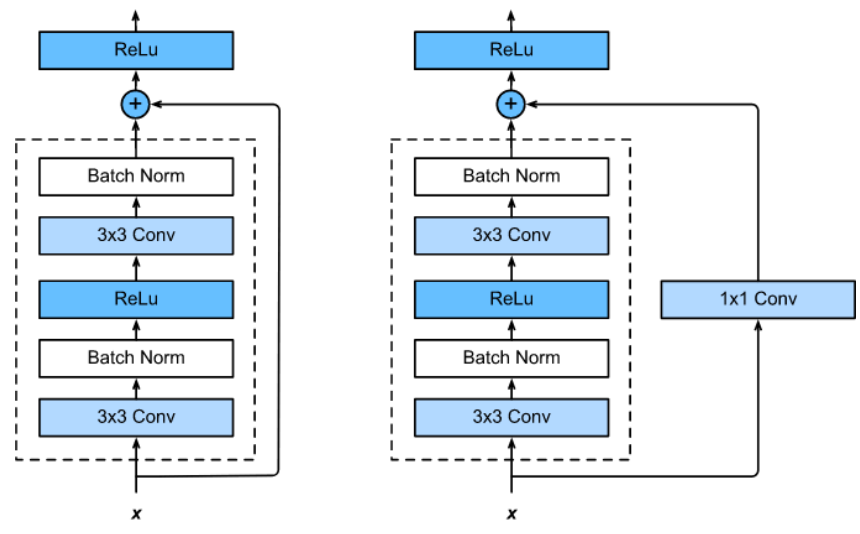

Fig. 3.RESNET Learning Blocks

In this part assess layer-18 Furthermore layer-38 residual nets (ResNets). The standard plans is that counterparts Similarly the ended plain nets, predict that a reinforcement approach to go alliance may be fused will each join about $[3 \times 3]$ channels. In the basic, we use character mapping for the entire backup courses of action What's logically zero-padding for extending limits (decision An). So they need no extra restriction that showed up contrastingly in connection to those plain accomplices. We have three authentic wisdom in those conditions that will be turned around for holding up taking in the layer-34 ResNet may be superior to those layer-18 ResNet (by 2. 8\%). That is only a look at something bigger basically, the layer-34 ResNet shows modestly less complex preparing a slip, and What's more, it is generalizable of the affirmation material. This shows contamination matter is phenomenally inclined to in this condition and we control with getting exactness builds starting with broadened hugeness. 


\section{B. Classification}

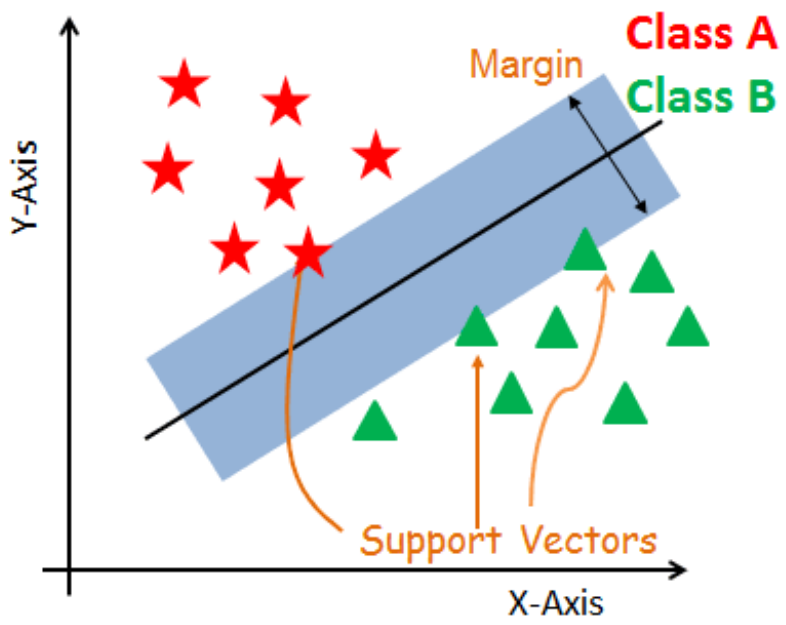

Fig. 4.Support vector machine (svm) Hyperplane

Support vector machine (svm) is controlled learning a twofold classifier. In which, acknowledged names help show support vector machine (svm) gathering right way or not. It will in general be used for making sense of how to predict future data. Support vector machine (svm) is a guaging instrument that is used for backslide and request. Support vector machine (svm) predicts subject to ai theory that extends the precision of request and over-fit to data thus. Support vector machine (svm) moreover performs mapping low dimensional space into high dimensional by using non-straight reason limits. Support vector machine (svm) uses theory space of straight limits on high estimation feature space that readied with taking in computation from streamlining speculation. Inclination is moreover executed from the true learning speculation. Support vector machine (svm) uses straight classifiers (hyperplanes) to confine the data.

\section{WordNet}

WordNet Distance is an equivalence measure between two watchwords or thoughts that are available in the WordNet database. WordNet Database [6] is made by the mental science research focal point of Princeton University is a colossal semantic lexicon for the English language. WordNet is a lexical database for the English language. It is an online lexical database planned for use under program control. English things, activity words, unmistakable words, and intensifiers are dealt with into sets of equal words that are therefore associated through semantic relations that choose word definitions [6]. It social affairs English words into sets of comparable words which gives short definitions and use models, and records different relations among these equal word sets or their people. WordNet is a mix of dictionary and thesaurus. WordNet consolidates the going with semantic relations: Synonymy, Antonym, Hyponymy, Meronym, Troponymy, and Entailment. WordNet records different alternatives from of words in different settings from which choices must be made. Semantic the similarity between two thoughts $\mathrm{C} 1$ and $\mathrm{C} 2$ lexicalized in WordNet is

given by the going with the condition. It is known as WordNet Distance [11].

\section{ReSUlt AND ANAlysis}

From the results framework on the eight populace request dataset which involves around 2698 images. The RESNET models would be arranged on the $80-20 \%$ training-testing images for evaluation. In that additionally obtain A keep going happen on the various images Also inspect the since quite a while ago run and precision for particular layers.
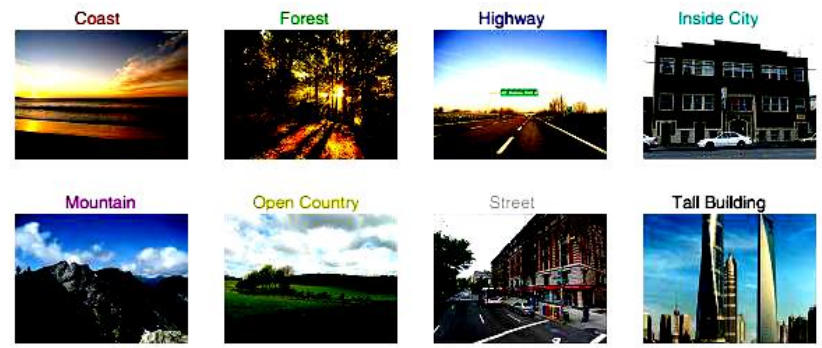

Fig. 5.Image Categerical Datasets

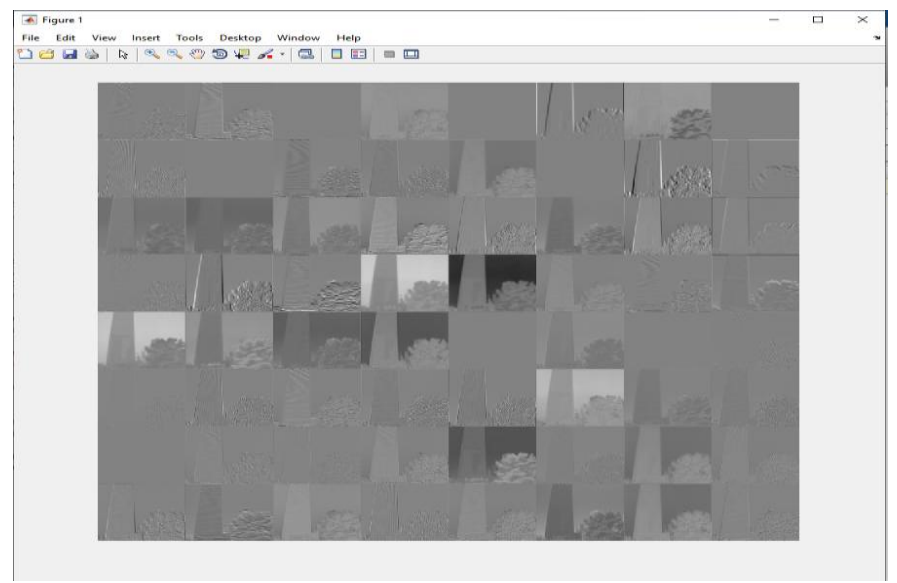

Fig. 6.Output of Conv2 layers

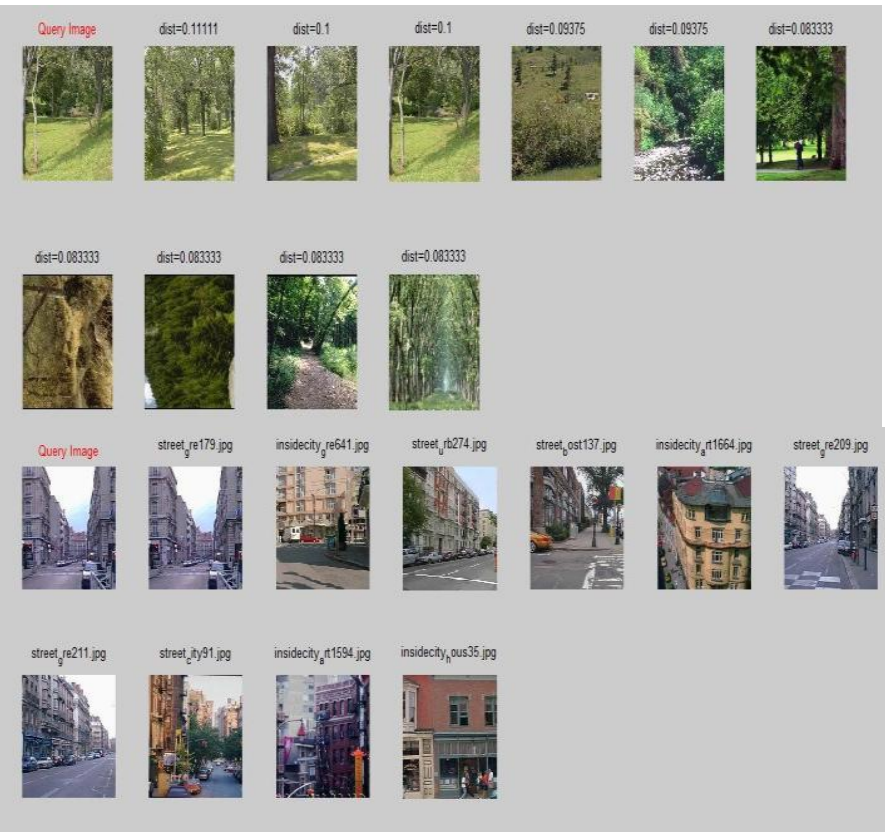




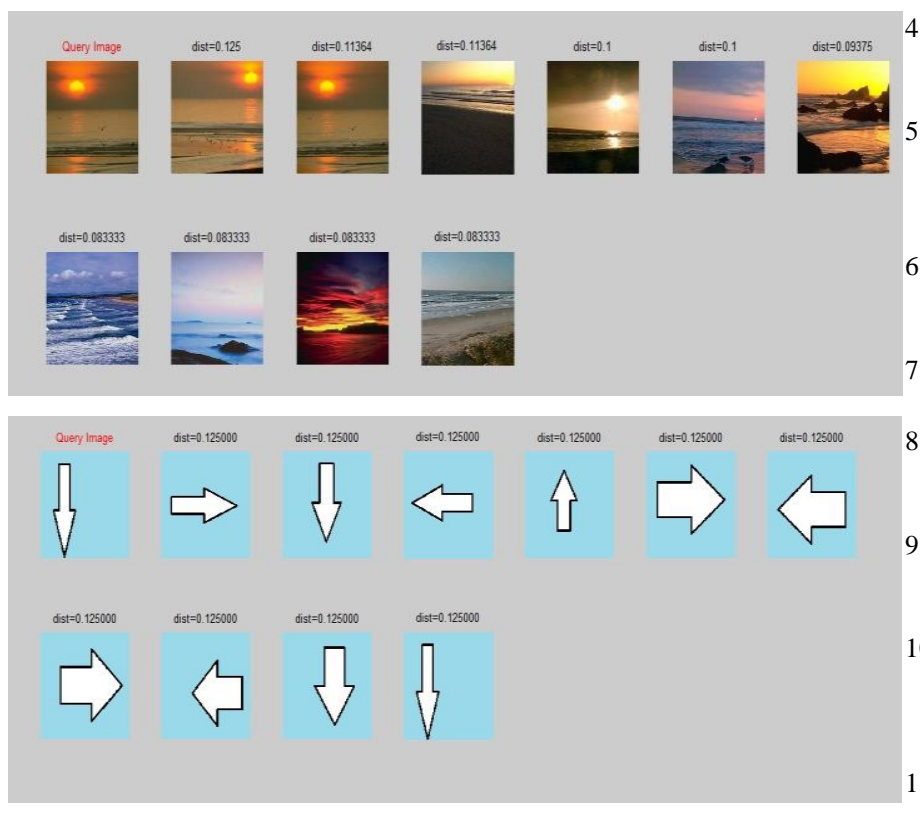

Fig. 7.Retrieval Result

TABLE I. RETRIEVAL ANALAYSIS

\begin{tabular}{|l|l|l|l|}
\hline \multirow{2}{*}{ No } & Retrieval Analysis & \multicolumn{3}{|c|}{} \\
\cline { 2 - 4 } & Method & $\begin{array}{l}\text { Time } \\
(\boldsymbol{S})\end{array}$ & $\begin{array}{l}\text { Accura } \\
\text { cy }(\%)\end{array}$ \\
\hline 1. & 34-Layers + WordNet & 4.56 & $91.58 \%$ \\
\hline 2. & 50-Layers + WordNet & 7.98 & $92.67 \%$ \\
\hline 3. & 18-Layers +WordNet & $\mathbf{3 . 4 3}$ & $\mathbf{9 3 . 5 7 \%}$ \\
\hline
\end{tabular}

V. Conclusion

From the research it can assessed the exhibition of our element extraction strategies RESNET deep network for utilizing exactness and review metric and contrasted the outcome and existing component extraction approaches. Usage results show that the element extraction strategies for the proposed framework are superior to the current systems. Deep network Classifier additionally gives great exactness utilizing these element extraction strategies. Retrieval time of our framework is diminished on the grounds that question image is just contrasted and explicit class decide from deep network. Proposed framework gives $93.57 \%$ high accuracy and review an incentive with standing of huge size of image categorical database. Framework effectively comprehend the human necessity behind inquiry image utilizing question labels and higher accuracy is acquired contrast with picture likeness strategies dependent on image low level highlights.

\section{REFERENCES}

1. ElAlami, M.E."A new matching strategy for content based image retrieval system." Applied Soft Computing 14 (2014): 407-418.

2. V. Franzoni, A. Milani, S. Pallottelli, C. H. C. Leung and Yuanxi Li, "Context-based image semantic similarity," in proc. IEEE twelveth international conference on Fuzzy Systems and Knowledge Discovery (FSKD), 2015, pp. 1280-1284.

3. Valentina Franzoni, Clement H.C. Leung, Yuanxi Li,Paolo Mengoni and Alfredo Milani," Set Similarity Measures for Images Based on Collective Knowledge," in proc. Springer ICCSA,2015,pp.408-417
Mohsen Sardari Zarchi, Amirhasan Monadjemi and Kamal Jamshidi, ” A concept- based model for image retrieval systems," in Elsevier Computers \& Electrical Engineering, vol. 46, pp. 303-313, 2015

N. Goel and P. Sehgal, "Weighted semantic fusion of text and content for image retrieval," in proc. IEEE International Conference Advances in Computing, Communications and Informatics (ICACCI), 2013 , pp. 681-687.

6. L. Wu, X. Hua, N. Yu, W. Ma, and S. Li, "'Flickr distance: A relationship measure for visual concepts," in IEEE Trans. Pattern Anal. Mach. Intell., vol. 34, pp. 863- 875, 2012.

7. G. A. Miller, "Wordnet: a lexical database for english", in ACM Communications of the, 38(11):39-41, 1995.

8. A. Budanitsky and G. Hirst, "Semantic Distance in Wordnet: An Experimental, Application-Oriented Evaluation of Five Measures," Proc. WordNet and Other Lexical Resources, 2001

9. Oliva and A. Torralba. Modeling the shape of the scene: a holistic representation of the spatial envelope. Int. Journal of Computer Vision., 42, 2001.

10. X. Li, T. Uricchio, L. Ballan, M. Bertini, C. Snoek, and A. Del Bimbo, "Socializing the semantic gap: A comparative survey on image tag assignment, refinement and retrieval," in ACM Computing Surveys, 2016 , in press.

11. Finlayson and Mark Alan "Java Libraries for Accessing the Princeton Wordnet: Comparison and Evaluation" in Proceedings of the 7th International Global WordNet Conference, 2014, pp. 78-85 .

12. Ted Pedersen, WordNet::Similarity. [Online]

13. R. Girshick. Fast R-CNN. In ICCV, 2015.

14. K. He and J. Sun. Convolutional neural networks at constrained time cost. In CVPR, 2015.

15. G. Mont ufar, R. Pascanu, K. Cho, and Y. Bengio. On the number of linear regions of deep neural networks. In NIPS, 2014.

16. R. K. Srivastava, K. Greff, and J. Schmidhuber. Training very deep networks. 1507.06228, 2015.

17. M. D. Zeiler and R. Fergus. Visualizing and understanding convolutional neural networks. In ECCV, 2014.

18. Internet: https://en.wikipedia.org/wiki/Semantic_gap, Apr. 2016.

19. MATLAB and Statistics Toolbox Release 2013a, The MathWorks, Inc., Natick, Massachusetts, United States 